Bulletin d'Histoire Contemporaine de l'Espagne

$51 \mid 2017$

Les forces politiques durant la Seconde République espagnole

\title{
La réception des Ballets russes à Madrid et Barcelone (1916-1929)
}

Hélène Frison

\section{OpenEdition}

\section{Journals}

Édition électronique

URL : http://journals.openedition.org/bhce/780

DOI : $10.4000 /$ bhce.780

ISSN : 1968-3723

Éditeur

Presses Universitaires de Provence

Édition imprimée

Date de publication : 1 juin 2017

Pagination : 284-287

ISSN : 0987-4135

Référence électronique

Hélène Frison, «La réception des Ballets russes à Madrid et Barcelone (1916-1929) », Bulletin d'Histoire Contemporaine de l'Espagne [En ligne], 51 | 2017, mis en ligne le 09 octobre 2018, consulté le 23 septembre 2020. URL : http://journals.openedition.org/bhce/780 ; DOI : https://doi.org/10.4000/bhce. 780 
republicana terminó por desangrar a la propia República. El 28 de marzo de 1939, las fuerzas franquistas entraban en Madrid. El 30 de marzo, la columna Sagardía ocupaba Alcalá de Henares.

La Guerra había terminado, y con ella la experiencia de más de setenta años de obrerismo en España y Alcalá. La represión contra el mismo no conoció precedentes. La oposición al franquismo fue amplia pero infructuosa. Había que esperar muchos años para que los trabajadores volvieran a dotarse de mecanismo de resistencia y lucha. Pero los referentes de la lucha habían pasado al exilio, a la cárcel o habían sido ejecutados por un régimen que se regodeó en su victoria y no tuvo piedad.

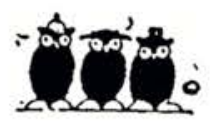

\section{La réception des Ballets russes à Madrid et Barcelone (1916-1929) ${ }^{1}$}

\section{Hélène FRISON}

La compagnie des Ballets russes, fondée par Diaghilev en 1911, constitue un tournant dans l'histoire de la scène occidentale. Reprenant le principe de l'œuvre d'art totale - rendu célèbre par la théorie du Gesamtkunstwerk de Wagner -, la troupe propose des spectacles composés par des peintres, des chorégraphes et des musiciens. Son succès est fulgurant et son influence décisive. Alors que l'Europe est en guerre, l'Espagne constitue une terre d'accueil propice aux échanges. Les

1 Thèse d'Études Hispaniques et Latinoaméricaines, soutenue à l'Université de Paris III, le 29 novembre 2014. Dir:: M. le prof. Serge Salaún. Jury: MM. et $\mathrm{M}^{\text {me }}$. les profs. Paul Aubert, président (Aix-Marseille Université), Serge Salaün, Manuel Aznar Soler (Univ. Autónoma, Barcelone), Eliseo Trenc (Univ. de Champagne), Evelyne Ricci (Univ. de Paris III). intellectuels du pays s'interrogent sur les possibilités de rénover la scène théâtrale et sont attentifs aux expériences qui viennent de l'étranger. Les ballets de la compagnie entrent en résonance avec leurs propres préoccupations: ils posent à la fois la question de la tradition au sein de la modernité, de la dimension nationale à visée internationale et s'exportent à l'étranger.

Malgré le nombre important de saisons données en Espagne, la Péninsule est le plus souvent absente des études consacrées aux Ballets russes. Les rares ouvrages qui l'évoquent s'intéressent principalement aux échanges qui ont eu lieu entre la troupe et les artistes espagnols. La perspective choisie dans cette thèse est autre: nous nous sommes proposée d'étudier l'influence qu'ont eue les Ballets sur leur pays d'accueil afin d'entrevoir, non une réception unique, mais une série de lectures, parfois divergentes, menées à un moment donné, par des intellectuels et artistes espagnols. À travers la compagnie, ce sont tour à tour les interrogations liées au renouvellement de la scène, à la constitution d'un langage artistique national et à la participation du pays à la modernité européenne qui sont posées. En d'autres termes, la compagnie constitue un révélateur des problématiques esthétiques, culturelles, sociales et idéologiques de l'Espagne des années folles.

Les Ballets russes constituent un événement singulier de la vie artistique espagnole des années 1910-1930. De l'ampleur de cette compagnie itinérante à la nature des spectacles présentés, ce sont bien l'ensemble de ses éléments qui détonnent au sein du panorama de la scène espagnole. Pourtant, ces ballets composés par des artistes de la modernité font salle comble. Réfutant le peu de succès rencontré jusque-là par les différentes expériences novatrices menées dans le pays, la compagnie réussit non seulement la prouesse de rassembler de nombreux spectateurs mais également celle de les 
fidéliser. L'un des principaux symptômes de la crise du théâtre espagnol résidait dans la dichotomie opposant les spectacles à succès aux tentatives de rénovation. Alors que les premiers attiraient les foules en essuyant le mépris des spécialistes de la scène, les secondes peinaient à sortir de l'extrême confidentialité. Les Ballets russes transcendent cette opposition: autour d'eux se pressent aussi bien les intellectuels promoteurs de l'avant-garde que la haute bourgeoisie espagnole. Et les artistes les moins académiques du pays investissent les salles traditionnellement réservées à la seule bonne société. Ce ralliement constitue sans doute la première réussite de la troupe: abolir l'opposition séculaire qui existe en Espagne entre les spectacles commerciaux et artistiques.

Une autre singularité caractérise la réception espagnole:les Ballets seproduisent tour à tour à Madrid et à Barcelone. Cette " ubiquité » permanente permet de mettre en évidence les personnalités des deux capitales culturelles du pays ainsi que les particularités de leur positionnement face à la modernité européenne.

À Madrid le succès est immédiat. Soutenue par les élites sociales et politiques, la troupe bénéficie de conditions de production particulièrement avantageuses. Elle est invitée à s'exhiber à plusieurs reprises et se produit systématiquement à guichet fermé. Largement relayée par la presse, la notoriété internationale des personnalités de la troupe participe également à l'engouement madrilène. Ce battage médiatique témoigne de la montée en puissance de la culture de masse: par l'écho qu'elle donne à l'événement, la presse réussit à inclure des individus qui n'y participent pas directement. Non seulement les journaux multiplient les articles sur les Ballets, mais ils proposent de nombreuses illustrations qui donnent à voir les spectacles: reproductions d'esquisses originales, photographies des personnages et portraits des danseurs, croquis de l'occupation de la scène. L'impact de cette diffusion est d'autant plus important que l'ensemble de la presse, des revues illustrées aux quotidiens, y participe.

Parallèlement à ce succès social et mondain, Diaghilev et ses collaborateurs sont fréquemment invités dans les cercles artistiques de la capitale. Cette réception, plus implicite que la précédente, est primordiale en ce qui concerne les liens qui se tissent entre les artistes. L'originalité de ces rencontres tient à la diversité des personnalités qui s'y croisent: les Ballets interpellent aussi bien les professionnels de la scène, que les musiciens et les intellectuels. Cette fascination s'explique par plusieurs raisons. D'une part, les corps athlétiques présents sur scène annoncent la naissance d'une culture moderne du sport; ils incarnent parfaitement la jeunesse et le sang neuf qu'Ortega y Gasset et Salazar appellent de leurs vœux. D'autre part, les Ballets constituent la symbiose la plus aboutie entre le national et le cosmopolitisme, et prouvent que brillamment adapté, le folklore peut s'exporter à l'international.

La présence de Falla dans la capitale joue également un rôle déterminant. Récemment rentré de son séjour parisien, le compositeur - soutenu par le critique Adolfo Salazar joue un rôle de «passeur» de la modernité entre les milieux avant-gardistes français et ceux artistiques espagnols. En outre, la collaboration de Falla avec la compagnie s'effectue au moment où le magistère du compositeur s'établit. Tout en définissant une voie esthétique - celle de la stylisation du folklore - le compositeur participe à la revalorisation du lien, conflictuel en Espagne, entre la scène et la musique symphonique. Dès lors que la danse cesse d'être une pratique sclérosée, dès lors qu'il existe en Espagne des danseurs susceptibles de porter le projet de modernité espéré par les compositeurs, l'écriture de ballet devient une troisième voie offerte à l'élaboration d'une musique nationale. 
Le Grupo de los Ocho, qui réunit la jeune génération incarnant la musique nouvelle à Madrid, témoigne de ce changement de considération ${ }^{2}$. Élaborant leur propre langage musical, ces compositeurs qui se revendiquent de Falla et de Salazar, du Groupe des Six et de Stravinsky, écrivent sans complexe ni réserve des partitions pour le ballet. Depuis la fin des années 1920 jusqu'à la guerre civile, le ballet devient, dans leur répertoire, un genre musical qui a toute sa place, au même titre que les œuvres symphoniques ou celles de musique de chambre.

Dans la capitale catalane, la réception emprunte une voie oblique. Pendant que Madrid poursuit sa quête de l'essence immémoriale du pays, Barcelone tourne ses regards vers l'extérieur pour mieux affirmer sa singularité. La ville revendique sa différence par son identification absolue à la modernité. Les yeux rivés sur Paris, Barcelone ne s'intéresse guère à la place de la tradition au sein des Ballets. Mais, contrairement à ce que laissait supposer l'exception catalane, la réception initiale de la troupe s'avère décevante par sa tiédeur. Cette réaction révèle en creux le strict cloisonnement qui sépare les milieux bourgeois de ceux artistiques. Alors que le Liceo fait systématiquement salle comble, les cercles les plus avant-gardistes de la ville maintiennent leur distance avec la troupe. Les ballets montés au Liceo sont cantonnés aux milieux bourgeois et les quelques infiltrations qui apparaissent, restent ponctuelles et circonscrites.

Il faut attendre la fin des années 1920 pour dépasser ce clivage à assister à

2 Le Grupo de los Ocho, parfois nommé également Grupo de Madrid, réunissait les musiciens suivants: les frères Ernesto (19051989) et Rodolfo (1900-1987) Halffter, Juan José Mantecón (1895-1964), Julián Bautista (1901-1961), Fernando Remacha (1898-1984), Rosa Garcia Ascot (1902-2002), Salvador Bacarrise (1898-1963) et Gustavo Pittaluga (1906-1975). la réhabilitation des Ballets. Dans le prolongement des saisons russes qui se renouvellent au fil des années, d'autres compagnies intègrent Barcelone à leurs circuits. Cette augmentation significative des spectacles de danse témoigne du changement d'horizon qui s'opère: la danse est peu à peu revalorisée au sein des arts vivants. Celle-ci a dorénavant son public et n'a plus besoin d'être accompagnée pour exister. Cette affirmation prend deux aspects: d'une part, le ballet néoclassique, notamment par la figure de Joan Magrinyà, acquiert ses lettres de noblesse sur la scène catalane; d'autre part, une pratique critique spécialisée s'initie sous l'impulsion de Sebastià Gasch. Ce changement qui s'enracine au tournant des années 1930, assure une existence posthume aux Ballets. Tour à tour vantée comme l'origine de ce mouvement rénovateur ou posée en mètre-étalon à l'aune duquel toute danse est désormais évaluée, la compagnie de Diaghilev devient une référence incontournable.

Partant du concept jaussien selon lequel une œuvre nouvelle dialogue nécessairement avec l'horizon d'attente dans lequel elle s'inscrit, la première partie de cette thèse $s$ 'attache à dresser un panorama de la scène espagnole contemporaine des saisons russes afin de mettre en lumière leurs facteurs différentiels. Ce tour d'horizon s'ouvre sur la réception des œuvres de Wagner à Madrid et à Barcelone car celles-ci constituent alors la référence artistique à laquelle sont systématiquement comparés les spectacles russes. Leur réception contrastée permet d'observer la lecture différenciée d'un même phénomène culturel. Un état des lieux des différentes pratiques théâtrales en vigueur succède à la présentation de la réception wagnérienne: arts de la scène, danse et musique, contemporains de la venue de Diaghilev sont ainsi exposés.

La réception de la première saison russe constitue le deuxième moment de cette étude. La première saison espagnole, 
comme tout phénomène nouveau, a placé le spectateur et le critique dans une position de découverte et a constitué un moment instaurateur. Comme le rappelle François Madurell, cette situation de découverte se distingue de celle de l'observation répétée et conditionne la perception que le spectateur a de l'œuvre ${ }^{3}$. Il semblait donc nécessaire de différencier les jugements esthétiques issus d'une situation de découverte à ceux portés en situation d'observation répétée. Plus que les autres, cette saison initiatrice semblait la plus à même de révéler l'écart esthétique perçu entre les nouveaux spectacles et l'expérience préalable du public, qui constituait alors son unique référence. Une radiographie des différentes réceptions est ainsi présentée afin de mettre en lumière l'altérité des Ballets aux yeux du spectateur espagnol, attentif à une autre esthétique plus conventionnelle.

À cette étude synchronique, succèdent les approches diachroniques des saisons madrilènes et barcelonaises. Ces troisième et quatrième parties se structurent de manière parallèle et analysent, après des pages consacrées à la description des ballets principaux et aux créations originelles, les débats qui sont apparus dans la presse et qui ont révélé des enjeux esthétiques et idéologiques spécifiques.

Le cas des deux villes a été étudié séparément car celles-ci présentent des contextes de production et des horizons d'attente différents. L'autonomie de lapresse catalane a été pour ce sujet déterminante car elle a permis de mettre en évidence les différences de réception en se fondant sur des centres de productions distincts. Partant de l'idée que le cadre de formulation,

3 François Madurell, «Situation de découverte et écoute répétée : deux clés pour la réception de l'œuvre ", in Anne-Marie Gouiffès, Emmanuel Reibel (coord.), Esthétique de la réception musicale, Actes de la Rencontre interartistique du 22 mars 2005, Paris, Observatoire Musical Français, 2007, p. 41-47. c'est-à-dire la nature du journal ainsi que sa ligne éditoriale, influe sur l'élaboration du jugement, un large corpus de recherche a été retenu. Sans chercher à analyser la totalité des articles publiés, nous avons choisi de retenir un ensemble représentatif: journaux quotidiens et périodiques, spécialisés et grand public, conservateurs et libéraux, laïcs et cléricaux. Ces nombreuses sources ont été étudiées tantôt par coupes verticales, tantôt par coupes horizontales, afin «d'allier à la continuité d'une diachronie la bigarrure de la synchronie $»^{4}$.

La présentation en miroir des deux villes met en évidence des réceptions différentes, inattendues et parfois contradictoires. Un point est cependant commun aux deux capitales culturelles espagnoles: l'enthousiasme des amateurs autant que des spécialistes face à ces spectacles d'exception qui sillonnent l'Europe:

«En 1917 (sic), définitivement déracinée de la patrie qui l'avait vu naître en raison de la révolution, la Compagnie des Ballets russes de Diaghilev étonnait le public du Teatro Real de Madrid, dès le lever du rideau. $[\ldots]$ Le prestige de son art avait davantage œuvré, lors de ses excursions à travers l'Europe, en faveur de la rénovation scénique que tous les exposés et polémiques qui avaient eu lieu $»^{5}$.

$4 \quad I d ., \mathrm{p}, 26$.

5 «En 1917 (sic), definitivamente desarraigada de su patria nativa por la revolución, la Compañia de Bailes Rusos del señor Diaghilef (sic) asombraba al público del Teatro Real de Madrid, no más levantarse el telón. [...] El prestigio de su arte habia hecho más en sus excursiones por Europa que todas las disquisiciones y polémicas en pro de la renovación escénica ", Cipriano de Rivas Cherif, Cómo hacer teatro: apuntes de orientación profesional en las artes y oficios del teatro español, Valencia, Pre-Textos, 1991, p. 40. 